\title{
Effect of orthodontic adhesive thickness on force required by debonding pliers
}

\author{
Tomohiko Hama1), Yasuhiro Namura ${ }^{1,2)}$, Yukina Nishio ${ }^{3)}$, \\ Takayuki Yoneyama ${ }^{4,5)}$, and Noriyoshi Shimizu ${ }^{1,2)}$
}

\author{
1)Department of Orthodontics, Nihon University School of Dentistry, Tokyo, Japan \\ 2)Division of Clinical Research, Dental Research Center, Nihon University School of Dentistry, Tokyo, Japan \\ 3)Division of Oral Structural and Functional Biology, Nihon University Graduate School of Dentistry, \\ Tokyo, Japan \\ 4)Department of Dental Materials, Nihon University School of Dentistry, Tokyo, Japan \\ ${ }^{5)}$ Division of Biomaterials Science, Dental Research Center, Nihon University School of Dentistry, \\ Tokyo, Japan
}

(Received February 12, 2014; Accepted May 22, 2014)

\begin{abstract}
This in vitro study evaluated the relationship between removal force and the thickness of three orthodontic adhesives, namely, light- and chemical-cured resin cements and a resin-modified glass ionomer cement. The thickness of each adhesive was $50,100,150$, or $200 \mu \mathrm{m}$, and all adhesives were bonded on bovine incisors. Removal force was measured before (TC-0) and after 1,000 thermal cycles (TC-1000), and values were compared. At TC-0, the removal strengths for adhesive thicknesses of 50 and $100 \mu \mathrm{m}$ were significantly lower than those for thicknesses of 150 and $200 \mu \mathrm{m}(P<0.05)$. At TC-1000, removal strengths for adhesive thicknesses of 50 and $100 \mu \mathrm{m}$ were also significantly lower than those for 150 and $200 \mu \mathrm{m}$. Superbond Orthomite specimens showed a significant difference in removal strength between TC-0 and TC-1000 $(P<0.05)$ at all thicknesses. There was no significant difference in the distribution of adhesive remnant index scores at any thickness. These findings indicate that decreasing the thickness of applied orthodontic adhesive reduces the
\end{abstract}

Correspondence to Dr. Yasuhiro Namura, Department of Orthodontics, Nihon University School of Dentistry, 1-8-13 Kanda-Surugadai, Chiyoda-ku, Tokyo 101-8310, Japan Fax: +81-3-3219-8312 E-mail: namura.yasuhiro@nihon-u.ac.jp

doi.org/10.2334/josnusd.56.185

DN/JST.JSTAGE/josnusd/56.185 removal strength required. (J Oral Sci 56, 185-190, 2014)

Keywords: removal strength; thickness; residual adhesive; thermal cycling.

\section{Introduction}

The maximum bond strength of orthodontic adhesives is around $25 \mathrm{MPa}$ (1-4), although bond strength depends on bonding conditions. Many reports (5-8) cite a study by Reynolds, which suggested that a bond strength of 6-8 $\mathrm{MPa}$ is required. Current adhesives exceed that strength and can withstand orthodontic treatment. However, because strongly bonded adhesive is ripped from the enamel surface with a bracket after treatment, enamel damage is a concern.

When a bonded bracket is removed, failure can occur at one of three interfaces: between the adhesive and enamel surface, within the bonding material itself, or between the adhesive cement and bracket. However, the interface between the adhesive cement and bracket is the usual site of failure when brackets are removed (9). Therefore, although the remaining adhesive must be removed, insufficient care during the removal process can damage the enamel surface. Additionally, the use of adhesive-removal pliers to remove adhesives may cause pain or enamel damage because of the large load applied to the teeth (9). 
Table 1 Materials used in this study

\begin{tabular}{|c|c|c|c|}
\hline Adhesive system & Chemical composition & Lot no. & Manufacturer \\
\hline Transbond XT (TX) & & & 3M Unitek \\
\hline Etching gel & $35 \%$ phosphoric acid & $5 \mathrm{FT}$ & (Monrovia, CA, USA) \\
\hline Light cure adhesive primer & Bis-GMA, TEGDMA & $712-034$ & \\
\hline Light cure adhesive paste & $\begin{array}{l}\text { Bis-GMA, silane-treated quartz, bisphenol A bis(2-hydroxy- } \\
\text { ethyl ether) dimethacrylate, silane-treated silica }\end{array}$ & 293905 & \\
\hline Superbond Orthomite (SB) & & & Sun Medical \\
\hline Red activator & $65 \%$ phosphoric acid & VG4 & (Moriyama, Japan) \\
\hline Monomer & MMA, 4-META & EM1 & \\
\hline Catalyst & TBB-O & VW51F & \\
\hline Polymer & PMMA & EG11 & \\
\hline Ortholy Glass Bond (OG) & & & $\mathrm{GC}$ \\
\hline A-paste & Fluoro-alumino-silicate glass, dimethacrylate, etc & 1103291 & (Tokyo, Japan) \\
\hline B-paste & Polyacrylic acid, silicon dioxide, distilled water, etc & 1103291 & \\
\hline Gel conditioner & Polyacrylic acid, distilled water, silicon dioxide, glycerin, etc & 0910161 & \\
\hline
\end{tabular}

Bis-GMA: bisphenol A diglycidyl ether dimethadrylate, TEGDMA: triethylene glycol dimethacrylate, MMA: methyl methacrylate, 4-META: 4-methacryloxyethyl trimellitate anhydride, TBB-O: partially oxidized tri- $n$-butylborane, PMMA: methyl methacrylate polymer

Although grinding the remaining adhesive with a carbide bar is the optimal approach (9), it is slow and inefficient (10). Therefore, if the load applied by debonding pliers could be reduced, most of the remaining resin could be removed without pain. Mehl et al. (11) reported that the thickness of the cement film affected the retentive strength of cementation in implant-retained crowns. We hypothesized that altering the thickness of the adhesive cement layer would reduce retentive strength (ie, the removal force required). Therefore, in this in vitro study we evaluated the relationship between the removal force required during removal of remnant adhesive cement and the thickness of orthodontic adhesives.

\section{Materials and Methods}

\section{Materials used}

The three orthodontic adhesives used in this study were a light-cured composite resin cement (TX; Transbond XT, 3M Unitek, Monrovia, CA, USA), 4-META/MMA-TBB resin (SB; Superbond Orthomite, Sun Medical, Moriyama, Japan), and a resin-modified glass ionomer cement (OG; Ortholy Glass Bond, GC, Tokyo, Japan), as shown in Table 1. All orthodontic adhesive systems were used according to the respective manufacturer's instructions. A curing unit (Optilux 501, SDS Kerr, Danbury, CT, USA; light intensity, $800 \mathrm{~mW} / \mathrm{cm}^{2}$ ) was also used.

\section{Tooth specimens}

Freshly extracted bovine permanent mandibular incisors were collected from a slaughterhouse. The criteria for tooth selection were intact labial enamel (ie, absence of fractures caused by extraction) and absence of caries.
Tooth specimens were stored at $-30^{\circ} \mathrm{C}$ until use.

Soft tissues were removed from each tooth. After the crown was separated from the root, the pulp was extirpated and the crown was stored in distilled water at room temperature until further use. The crown was then embedded in self-curing acrylic resin (Tray Resin, Shofu, Kyoto, Japan) to facilitate its placement in the testing machine. The labial surface of each crown was flattened to facilitate force application and polished with waterproof silicon-carbide papers (\#400, \#600). The enamel surfaces were rinsed with water and dried in an oil-free airstream.

\section{Surface treatment procedure}

For application of TX adhesive, the enamel surface was etched with 35\% phosphoric acid gel (Transbond XT Etching Gel, 3M Unitek) for $30 \mathrm{~s}$, washed for $20 \mathrm{~s}$, and then dried with oil-free compressed air. The bonding agent (Transbond XT Light Cure Adhesive Primer, 3M Unitek) was applied to the treated surface.

For application of SB adhesive, the enamel surface was etched with red activator for $30 \mathrm{~s}$, washed for $20 \mathrm{~s}$, and then dried with oil-free compressed air. A partially oxidized tri- $n$-butylborane (TBB) catalyst was added to the monomer liquid, and the polymer and activated monomer liquid were then mixed.

For application of OG adhesive, the gel conditioner was applied to the enamel surface, left for $20 \mathrm{~s}$, washed for $20 \mathrm{~s}$, and then dried with oil-free compressed air. The $\mathrm{A}$ and $\mathrm{B}$ pastes were then mixed for application to the enamel surface. 


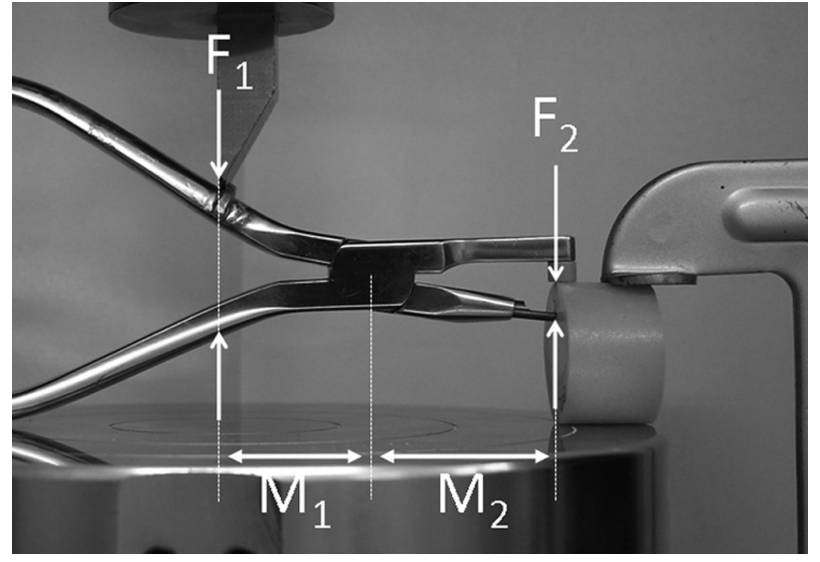

Fig. 1 Method for applying removal force.

\section{Establishing adhesive thickness}

The thickness of the adhesive layer was set to 50, 100, 150 , or $200 \mu \mathrm{m}$ for each adhesive. Masking tape that had been piled up to the required thickness and punched with a hole (diameter, $2 \mathrm{~mm}$ ) was applied to the enamel, the surface of which had been treated as described above. The hole was filled with the respective adhesive paste and mixtures, and the outer surface was covered with a polyester matrix tape (3M ESPE, St Paul, MN, USA). Light curing was carried out for $30 \mathrm{~s}$ through the polyester-matrix tape, except for the SB adhesive, which was chemical-cured. After light curing and initial curing, waterproof silicon-carbide papers were used to adjust the thickness until it was as described above. Thickness was confirmed with a slide caliper (NTD12P-15, Mitutoyo, Kawasaki, Japan).

\section{Thermal cycling}

To investigate the effects of thermal changes on adhesive durability, the specimens were subjected to continuous thermal cycling for 1,000 cycles between $4{ }^{\circ} \mathrm{C}$ and $60^{\circ} \mathrm{C}$ water baths, with a 30 -s dwell time in each bath, after storing for $24 \mathrm{~h}$ in distilled water at $37^{\circ} \mathrm{C}$.

\section{Measurement of removal force}

A universal testing machine (5567, Instron, Norwood, MA, USA) was used to measure the force required to remove the adhesive layer (Fig. 1) at a crosshead speed of $1 \mathrm{~mm} / \mathrm{min}$. Adhesives - which had been stored for $24 \mathrm{~h}$ in distilled water at $37^{\circ} \mathrm{C}$ (TC-0) or subjected to thermal cycling (TC-1000)-were removed using adhesive-removal pliers (802-1006, Tomy, Tokyo, Japan) according to the modified method of Bishara et al. (12) and Horiuchi et al. (13). The steel blade tip of the pliers was placed at the adhesive-enamel interface, and a squeezing action was applied until bond failure occurred. The relationship between the measured failure load, $\mathrm{F}_{1}$, and the removal force, $F_{2}$, is $F_{1} M_{1}=F_{2} M_{2}$. Thus, $F_{2}=$ $F_{1} M_{1} / M_{2}$, where $M_{1}$ and $M_{2}$ are the moment arms in Fig. 1. To calculate removal strength the removal force, $\mathrm{F}_{2}$, in Newtons was divided by $3.14 \mathrm{~mm}^{2}$ (the cross-sectional area of the adhesive bonding surface).

\section{Assessment of residual adhesive}

After calculating removal strength, each specimen was examined under an optical microscope (SZ-3003; As one, Osaka, Japan) at $15 \times$ magnification to identify the fracture pattern of the bonded surface. Residual adhesive on each tooth was assessed using the modified adhesive remnant index (ARI), as follows: 0, no adhesive remaining; 1 , $<50 \%$ adhesive remaining; $2, \geq 50 \%$ adhesive remaining; $3,100 \%$ adhesive remaining (14).

\section{Statistical analysis}

All data were statistically analyzed, and descriptive statistics, including the mean and standard deviation (SD), were calculated for each group by using the SPSS software package (SPSS Inc., Chicago, IL, USA). In addition, the Kolmogorov-Smirnov and Levene tests were used to verify the normality and homogeneity of variance. Before and after each thermal cycling test, differences among groups were evaluated using the Games-Howell test. Differences between values before and after thermocycling were analyzed with Welch's $t$-test. Differences in the distribution of ARI scores were analyzed using the chi-square test. A $P$ value less than 0.05 was considered to indicate statistical significance.

\section{Results}

The removal strengths of each adhesive are listed in Table 2. For TX, SB, and OG, removal strengths were significantly lower at thicknesses of 50 and $100 \mu \mathrm{m}$ than at thicknesses of 150 and $200 \mu \mathrm{m}(P<0.05)$. The ARI scores for each adhesive are shown in Table 3. Chi-square analysis revealed no significant differences in ARI score distribution at any adhesive thickness. However, TX specimens with an adhesive thickness of $150 \mu \mathrm{m}$ were more likely to have no remnant adhesive on the tooth (ARI score, 0), as compared with other thicknesses. SB specimens with adhesive thicknesses of 100 and $150 \mu \mathrm{m}$ were also more likely to have no remnant adhesive on the tooth (ARI score, 0 ), as compared with other thicknesses.

The removal strengths of each adhesive after thermocycling are shown in Table 4. In the TX group, removal strengths were significantly greater at thicknesses of 150 and $200 \mu \mathrm{m}$ than at thicknesses of 50 and $100 \mu \mathrm{m}$. In 
Table 2 Removal strength (MPa) in relation to adhesive thickness, at 0 thermal cycles

\begin{tabular}{lllcc}
\hline & \multicolumn{4}{c}{ Thickness $(\mu \mathrm{m})$} \\
Adhesive & 50 & 100 & 150 & 200 \\
\hline TX & $7.6^{\text {acd }}$ & $9.4^{\text {ad }}$ & $13.3^{\mathrm{b}}$ & $15.9^{\mathrm{b}}$ \\
& $(3.2)$ & $(2.6)$ & $(1.2)$ & $(4.3)$ \\
SB & $8.9^{\text {ad }}$ & $9.7^{\mathrm{a}}$ & $13.3^{\mathrm{b}}$ & $17.4^{\mathrm{b}}$ \\
& $(2.6)$ & $(0.6)$ & $(1.8)$ & $(3.5)$ \\
OG & $4,1^{\mathrm{c}}$ & $4.4^{\mathrm{c}}$ & $6.8^{\mathrm{de}}$ & $8.0^{\mathrm{ae}}$ \\
& $(0.6)$ & $(0.8)$ & $(0.6)$ & $(2.4)$ \\
\hline
\end{tabular}

Identical superscript letters indicate nonsignificant differences $(P>0.05)$.

$n=10$; parentheses indicate standard deviation

Table 3 Adhesive remnant index (ARI) scores for each adhesive

\begin{tabular}{|c|c|c|c|c|c|}
\hline \multirow[b]{3}{*}{ Adhesive } & & \multicolumn{4}{|c|}{ Thickness $(\mu \mathrm{m})$} \\
\hline & & 50 & 100 & 150 & 200 \\
\hline & Score & $\begin{array}{llll}0 & 1 & 2 & 3\end{array}$ & $\begin{array}{llll}0 & 1 & 2 & 3\end{array}$ & $\begin{array}{llll}0 & 1 & 2 & 3 \\
\end{array}$ & $\begin{array}{llll}0 & 1 & 2 & 3 \\
\end{array}$ \\
\hline $\mathrm{TX}$ & & 46000 & 45110 & 28800 & $\begin{array}{llll}5 & 5 & 0 & 0\end{array}$ \\
\hline SB & & 73300 & $\begin{array}{llll}5 & 5 & 0 & 0\end{array}$ & $\begin{array}{llll}4 & 6 & 0 & 0\end{array}$ & 82200 \\
\hline OG & & 73000 & 73300 & 82200 & $9 \begin{array}{llll}9 & 0 & 0\end{array}$ \\
\hline
\end{tabular}

ARI scores: 0 , no adhesive left on tooth surface; $1,<50 \%$ of adhesive left on tooth surface; $2,>50 \%$ of adhesive left on tooth surface; 3 , adhesive left on tooth surface.

There was no significant difference $(P>0.05)$ in relation to thickness for any adhesive.

Table 4 Removal strength (MPa) in relation to adhesive thickness, at 1,000 thermal cycles

\begin{tabular}{lllcc}
\hline & \multicolumn{4}{c}{ Adhesive thickness $(\mu \mathrm{m})$} \\
Adhesive & 50 & 100 & 150 & 200 \\
\hline TX & $5.9^{\text {acgh }}$ & $8.8^{\text {bd }}$ & $12.7^{\mathrm{c}}$ & $13.7^{\mathrm{c}}$ \\
& $(1.8)$ & $(1.5)$ & $(1.2)$ & $(0.9)$ \\
SB & $6.1^{\mathrm{a}^{*}}$ & $6.7^{\mathrm{a}^{*}}$ & $7.9^{\text {bef* }^{*}}$ & $8.7^{\text {bै* }^{*}}$ \\
& $(0.6)$ & $(0.6)$ & $(0.3)$ & $(1.3)$ \\
OG & $3.7^{\mathrm{g}}$ & $3.9^{\mathrm{g}}$ & $4.7^{\mathrm{h}^{*}}$ & $6.5^{\mathrm{a}}$ \\
& $(0.3)$ & $(0.5)$ & $(0.4)$ & $(0.7)$ \\
\hline
\end{tabular}

Identical superscript letters indicate nonsignificant differences $(P>0.05)$.

Asterisks indicate a significant difference $(P<0.05)$ between 0 and 1,000 thermal cycles for the same adhesive and thickness.

$n=10$; parentheses indicate standard deviation

Table 5 Adhesive remnant index (ARI) scores for each adhesive, at 1,000 thermocycles

\begin{tabular}{|c|c|c|c|c|c|}
\hline \multirow[b]{3}{*}{ Adhesive } & & \multicolumn{4}{|c|}{ Thickness $(\mu \mathrm{m})$} \\
\hline & & 50 & 100 & 150 & 200 \\
\hline & Score & $\begin{array}{llll}0 & 1 & 2 & 3 \\
\end{array}$ & $\begin{array}{llll}0 & 1 & 2 & 3 \\
\end{array}$ & $\begin{array}{llll}0 & 1 & 2 & 3\end{array}$ & $\begin{array}{llll}0 & 1 & 2 & 3 \\
\end{array}$ \\
\hline TX & & 64000 & 64000 & $\begin{array}{llll}5 & 5 & 0 & 0\end{array}$ & $\begin{array}{llll}4 & 5 & 1 & 0\end{array}$ \\
\hline SB & & $\begin{array}{llll}5 & 5 & 0 & 0\end{array}$ & $\begin{array}{llll}10 & 0 & 0 & 0\end{array}$ & $\begin{array}{llll}10 & 0 & 0 & 0\end{array}$ & $\begin{array}{llll}10 & 0 & 0 & 0\end{array}$ \\
\hline OG & & $\begin{array}{llll}9 & 1 & 0 & 0\end{array}$ & $\begin{array}{llll}9 & 1 & 0 & 0\end{array}$ & $9 \begin{array}{llll}9 & 1 & 0 & 0\end{array}$ & $\begin{array}{llll}10 & 0 & 0 & 0\end{array}$ \\
\hline
\end{tabular}

ARI scores: 0 , no adhesive left on tooth surface; $1,<50 \%$ of adhesive left on tooth surface; $2,>50 \%$ of adhesive left on tooth surface; 3 , adhesive left on tooth surface.

There was no significant difference $(P>0.05)$ in relation to thickness for any adhesive.

the SB group, removal strengths were significantly lower at thicknesses of 50 and $100 \mu \mathrm{m}$ than at 150 and 200 $\mu \mathrm{m}(P<0.05)$. In the OG group, removal strengths were significantly lower at thicknesses of 50 and $100 \mu \mathrm{m}$ than at 150 and $200 \mu \mathrm{m}$. For all adhesive thicknesses in SB specimens, there was a significant difference in removal 
strength between TC-0 and TC-1000 specimens $(P<$ $0.05)$. In addition, there was a significant difference between TC-0 and TC-1000 in $150-\mu \mathrm{m}$ OG specimens (Table 4). The ARI scores for all adhesives at TC-1000 are listed in Table 5. In the SB group, the 50- $\mu$ m specimens were less likely to have no adhesive on the tooth (ARI score, 0 ) than were specimens with other adhesive thicknesses. However, there was no significant difference in ARI scores in relation to adhesive thickness for any adhesive.

\section{Discussion}

This study focused on the residual adhesive cement layer after debracketing, and demonstrated that removal strength was lower when the adhesive cement layer was thinner. Regarding the relationship between adhesive thickness and bond strength, Jost-Brinkmann et al. (15) reported that composites with macrofillers yielded the highest bond strengths and that the thickness of the adhesive layer had no effect. Mackay (16) reported that increasing the thickness of four adhesives had no significant effect on mean shear bond strengths, although strength tended to decrease. However, these previous experiments used a model in which the bracket was bonded onto the tooth. Therefore, a loaded shear force would be applied to the lateral surface of both the adhesive and bracket.

Mehl et al. (11) evaluated the effects of cement film thickness on retention of cemented implant-retained crowns and found that crown retention decreased significantly between cement films with thicknesses of 15 and $50 \mu \mathrm{m}$. Wakasa et al. (17) reported that during a shear bond test the change in average stress with interfacial stress was related to the thickness of the bonding area.

Regarding mechanics during loading, using threedimensional finite element analysis, Hatamleh et al. (18) found that shear stresses increased with increasing distance and bending moment upon application of a uniform shear load at different distances from the interface. Although the blade of the pliers contacts the side of remnant adhesive when removing adhesive, the load is more concentrated when the adhesive is thinner, and the bending moment and shear force at the tooth surface are lower. Adhesives of various thicknesses were compared in the present study; removal strengths were significantly lower for adhesive layer thicknesses of 50 and $100 \mu \mathrm{m}$ than for thicknesses of 150 and $200 \mu \mathrm{m}$. Our findings suggest that removal strength during adhesive removal can be significantly reduced, by $20 \%$ to $60 \%$, by decreasing adhesive thickness to $100 \mu \mathrm{m}$.

We also evaluated the effect of thermal cycling on removal strength. For all materials tested, removal strength tended to be higher after thermal cycling; post-thermocycling removal strengths for SB and $\mathrm{OG}$ specimens with a thickness of $150 \mu \mathrm{m}$ were significantly lower before thermocycling. The significant difference in SB specimens might be due to the physical properties of 4-META/MMA-TBB resin (19). However, there were no significant differences in the removal strength for TX specimens at TC-0 and TC-1000. Therefore, the thickness of TX should be reduced to decrease subsequent discomfort during removal.

The bonded area established in this experiment was equivalent to one-third of one bracket section. Several researchers have described residual adhesive levels after debracketing, at various bond strengths (20-23). Rikuta et al. (24) reported that an area of more than $50 \%$ of the SB, which was used to bond the metal bracket onto the tooth surface in a shear test, occurred in $90 \%$ of specimens. Algera et al. (25) reported that a remnant area of more than 50\% was common. Thus, regardless of frequency, the extent of adhesive application in this study matches that remaining on the tooth surface after debracketing under actual clinical conditions. We used adhesive thicknesses of 50,100,150, and $200 \mu \mathrm{m}$ because a previous study (26) reported that the thickness of residual adhesive on teeth was $102.7 \pm 80 \mu \mathrm{m}$ after debonding.

\section{Acknowledgments}

This work was supported, in part, by a grant in 2013 from the Dental Research Center of Nihon University School of Dentistry.

\section{References}

1. Coreil MN, McInnes-Ledoux P, Ledoux WR, Weinberg R (1990) Shear bond strength of four orthodontic bonding systems. Am J Orthod Dentofacial Orthop 97, 126-129.

2. Su J, Hobson RS, McCabe JF (2004) Effect of impression technique on bond strength. Am J Orthod Dentofacial Orthop $125,51-55$.

3. Samruajbenjakul B, Kukiattrakoon B (2009) Shear bond strength of ceramic brackets with different base designs to feldspathic porcelains. Angle Orthod 79, 571-576.

4. Sfondrini MF, Gatti S, Scribante A (2011) Shear bond strength of self-ligating brackets. Eur J Orthod 33, 71-74.

5. Bishara SE, VonWald L, Olsen ME, Laffoon JF (1999) Effect of time on the shear bond strength of glass ionomer and composite orthodontic adhesives. Am J Orthod Dentofacial Orthop 116, 616-620.

6. Bishara SE, Laffoon JF, VonWald L, Warren J (2002) Effects of time on the shear bond strength of cyanoacrylate and composite orthodontic adhesives. Am J Orthod Dentofacial Orthop 121, 297-300.

7. Liu JK, Chuang SF, Chang CY, Pan YJ (2004) Comparison of 
initial shear bond strengths of plastic and metal brackets. Eur J Orthod 26, 531-534.

8. Yamamoto A, Yoshida T, Tsubota K, Takamizawa T, Kurokawa H, Miyazaki M (2006) Orthodontic bracket bonding: enamel bond strength vs time. Am J Orthod Dentofacial Orthop 130, 435.e1-6.

9. Proffit WR (2013) The third stage of comprehensive treatment: finishing. In: Contemporary orthodontics, 5th ed, Proffit WR, Fields HW, Sarver DM eds, Mosby Elsevier, St Louis, 582-605.

10. Eliades T, Gioka C, Eliades G, Makou M (2004) Enamel surface roughness following debonding using two resin grinding methods. Eur J Orthod 26, 333-338.

11. Mehl C, Harder S, Steiner M, Vollrath O, Kern M (2013) Influence of cement film thickness on the retention of implant-retained crowns. J Prosthodont 22, 618-625.

12. Bishara SE, Fonseca JM, Boyer DB (1995) The use of debonding pliers in the removal of ceramic brackets: force levels and enamel cracks. Am J Orthod Dentofacial Orthop 108, 242-248.

13. Horiuchi S, Kaneko K, Mori H, Kawakami E, Tsukahara T, Yamamoto K et al. (2009) Enamel bonding of self-etching and phosphoric acid-etching orthodontic adhesives in simulated clinical conditions: debonding force and enamel surface. Dent Mater J 28, 419-425.

14. Årtun K, Bergland S (1984) Clinical trials with crystal growth conditioning as an alternative to acid-etch enamel pretreatment. Am J Orthod 85, 333-340.

15. Jost-Brinkmann PG, Schiffer A, Miethke RR (1992) The effect of adhesive-layer thickness on bond strength. J Clin Orthod 26, 718-720.

16. Mackay F (1992) The effect of adhesive type and thickness on bond strength of orthodontic brackets. Br J Orthod 19, 35-39.

17. Wakasa K, Yamaki M, Matsui A (1995) Calculation models for average stress and plastic deformation zone size of bonding area in dentine bonding systems. Dent Mater J 14, 152-165.

18. Hatamleh MM, Rodrigues FP, Silikas N, Watts DC (2011) 3D-FE analysis of soft liner-acrylic interfaces under shear loading. Dent Mater 27, 445-454.

19. Hayakawa T, Nemoto K (2002) Efficacy of self-etching primers in the adhesion of 4-META/MMA-TBB resin cement to enamel. J Adhes Dent 4, 105-113.

20. Arici S, Caniklioglu CM, Arici N, Ozer M, Oguz B (2005) Adhesive thickness effects on the bond strength of a lightcured resin-modified glass ionomer cement. Angle Orthod 75, 254-259.

21. Faltermeier A, Behr M, Müssig D (2007) A comparative evaluation of bracket bonding with 1-, 2-, and 3-component adhesive systems. Am J Orthod Dentofacial Orthop 132, 144. e1-5.

22. Iijima M, Ito S, Yuasa T, Muguruma T, Saito T, Mizoguchi I (2009) Effects of application time and agitation for bonding orthodontic brackets with two self-etching primer systems. Dent Mater J 28, 89-95.

23. Parrish BC, Katona TR, Isikbay SC, Stewart KT, Kula KS (2012) The effects of application time of a self-etching primer and debonding methods on bracket bond strength. Angle Orthod 82, 131-136.

24. Rikuta A, Yoshida T, Tsubota K, Tsuchiya H, Tsujimoto A, Ota $\mathrm{M}$ et al. (2008) Influence of environmental conditions on orthodontic bracket bonding of self-etching systems. Dent Mater J 27, 654-659.

25. Algera TJ, Kleverlaan CJ, Prahl-Andersen B, Feilzer AJ (2008) The influence of different bracket base surfaces on tensile and shear bond strength. Eur J Orthod 30, 490-494.

26. Al Shamsi AH, Cunningham JL, Lamey PJ, Lynch E (2007) Three-dimensional measurement of residual adhesive and enamel loss on teeth after debonding of orthodontic brackets: an in-vitro study. Am J Orthod Dentofacial Orthop 131, 301. e9-15. 